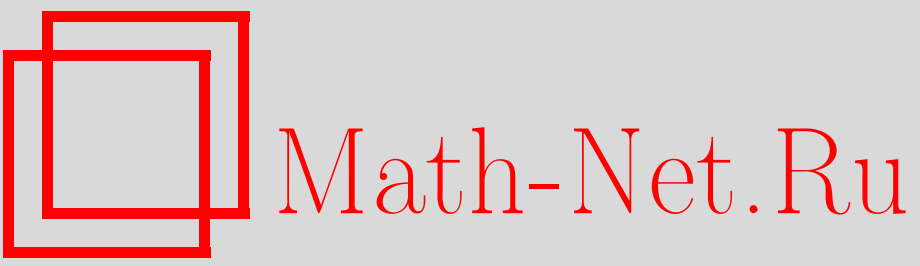

О. В. Самарина, С. П. Семенов, В. В. Славский, Анализ трехканальных изображений, основанный на теории три-тканей, Итоги науки и техн. Сер. Соврем. мат. и ее прил. Темат. обз., 2020, том 182, 119-124

DOI: https://doi.org/10.36535/0233-6723-2020-182-119-124

Использование Общероссийского математического портала Math-Net.Ru подразумевает, что вы прочитали и согласны с пользовательским соглашением

http://www.mathnet.ru/rus/agreement

Параметры загрузки:

IP : 54.197 .217 .227

26 апреля 2023 г., 13:01:14 


\title{
АНАЛИЗ ТРЕХКАНАЛЬНЫХ ИЗОБРАЖЕНИЙ, ОСНОВАННЫЙ НА ТЕОРИИ ТРИ-ТКАНЕЙ
}

\author{
(c) 2020 г. О. В. САМАРИНА, С. П. СЕМЕНОВ, В. В. СЛАВСКИЙ
}

\begin{abstract}
АннотАция. В настоящее время одной из наиболее актуальных задач цифровой обработки изображений является поиск новых математических подходов к анализу и обработке многоканальных изображений. Актуальность этих исследований обусловлена необходимостью оптимизации имеющихся методов цифровой обработки изображений, повышения эффективности и качества получаемых результатов. В работе предложен новый подход к обработке цифрового RGB-изображения, основанный на теории три-тканей В. Бляшке. Определяются и исследуются инварианты трехканальных изображений относительно максимально широкой «топологической» группы преобразований.
\end{abstract}

Ключевые слова: многоканальное изображение, три-ткань, инвариант.

\section{ANALYSIS OF THREE-CHANNEL IMAGES BASED ON THE THEORY OF THREE-WEBS}

\author{
(c) 2020 O. V. SAMARINA, S. P. SEMENOV, V. V. SLAVSKY
}

\begin{abstract}
Currently, one of the most urgent problems of digital image processing is the search for new mathematical approaches to the analysis and processing of multichannel images. The relevance of these studies is due to the need of optimization of available methods of digital image processing and increase the quality of the results obtained. In this paper, a new approach to processing of digital RGB-images based on the Blaschke theory of three-webs is proposed. Invariants of three-channel images with respect to the widest "topological" transformation group are determined.
\end{abstract}

Keywords and phrases: multichannel image, three-web, invariant.

AMS Subject Classification: 62H35, 65D18

1. Введение. Растровое монохромное изображение в математике представляют как прямоугольный двумерный массив чисел. При этом каждое число принимает целое значение в шкале $[0,255]$ и соответствует одному элементу изображения или пикселю. Для цветного трехканального RGB-изображения соответственно нужны три таких массива. Если отвлечься от технических деталей и не учитывать дискретность (пиксельное строение), то цифровое трехканальное RGBизображение задается в виде трех неотрицательных функций $u_{i}(x, y), i=1,2,3$, в некоторой области $D$ на плоскости.

С точностью до цветовой коррекции (переходу к другой шкале) такое изображение определяется семействами линий уровня функций $u_{i}(x, y), i=1,2,3$, где $u_{i}(x, y) \in C^{3}(D)$. Кроме того, часто изображение определено с точностью до некоторого диффеоморфизма области $D$. Если рассматривать наиболее широкий класс таких преобразований, то получится объект, который

Работа выполнена при поддержке Российского фонда фундаментальных исследований (проекты №o 18-47860016, 18-01-00620) и Научного Фонда Югорского государственного университета (проект № 13-01-20/10). 
был введен в конце 1920-х гг. выдающимся немецким геометром В. Бляшке под названием триткани (см. [2]).

Представим трехканальное изображение в виде трех неотрицательных функций $u_{i}(x, y), i=$ $1,2,3$, в некоторой области $D$ на плоскости. Необходимые свойства этих функций будут сформулированы позднее. Семейства линий уровня этих функций будут иметь вид

$$
L_{1}=\left\{(x, y): u_{1}(x, y)=\mathrm{const}\right\}, \quad L_{2}=\left\{(x, y): u_{2}(x, y)=\mathrm{const}\right\}, \quad L_{3}=\left\{(x, y): u_{3}(x, y)=\mathrm{const}\right\}
$$

Будем называть эти три семейства линий топографической сеткой или три-тканью данного изображения (см. $[1,7])$. Функцией три-ткани называется любая функция $W\left(u_{1}, u_{2}, u_{3}\right)$, не равная тождественно константе, для которой что в области $D$ выполняется тождество

$$
W\left(u_{1}(x, y), u_{2}(x, y), u_{3}(x, y)\right) \equiv 0 .
$$

Эта функция выражает зависимость, связывающую три функции в окрестности данной точки. При общих преобразованиях изображений, относящихся к группе гомеоморфизмов и имеющих вид

$$
x=\varphi_{1}\left(x^{\prime}, y^{\prime}\right), \quad y=\varphi_{2}\left(x^{\prime}, y^{\prime}\right)
$$

функция три-ткани не меняется и, следовательно, является «топологическим инвариантом изображения».

Замечание 1. Исследование функции три-ткани особенно полезно при решении задач дистанционного зондирования поверхности Земли. Спутниковые снимки, как правило, многоканальные, и знание корреляции между интенсивностью каналов может дать лишь первую грубую оценку зависимости между каналами. Функция три-тканей позволяет учесть не только интенсивность каналов, но и более тонкие зависимости, основанные на использовании теории распознавания образов.

\section{2. Инвариантные характеристики три-ткани RGB-изображения.}

2.1. Каноническое представление функиии RGB-изображения как наиболее общий инвариант. Наиболее современное изложение теории три-тканей можно найти в [1,5]. Далее мы будем придерживаться менее формального первоначального изложения теории В. Бляшке в его работе «Введение в геометрию тканей». В этой работе он определил каноническое представление функции три-ткани как наиболее общий инвариант функции три-ткани. Рассмотрим данный инвариант три-ткани изображения подробнее.

Предположим, что в точке $P_{0}=\left(x_{0}, y_{0}\right)$ выполняется условие

$$
u_{1}\left(P_{0}\right)=u_{2}\left(P_{0}\right)=u_{3}\left(P_{0}\right)=0 .
$$

Разложим функцию ткани в степенной ряд по $\left\{u_{1}, u_{2}, u_{3}\right\}$ :

$$
W=W_{1} u_{1}+W_{2} u_{2}+W_{3} u_{3}+\frac{1}{2} W_{1} u_{1}^{2}+W_{12} u_{1} u_{2}+\ldots
$$

Здесь величины $W_{i}, W_{i j}, \ldots$ - частные производные первого, второго порядка функции ткани $W$ по переменным $u_{i}$. Для функции $W$ определены преобразования следующего вида (см. [2]):

$$
\text { I : } W^{*}\left(u_{1}^{*}, u_{2}^{*}, u_{3}^{*}\right)=W\left[u_{1}\left(u_{1}^{*}\right), u_{2}\left(u_{2}^{*}\right), u_{3}\left(u_{3}^{*}\right)\right]
$$

- замена параметра в каждом семействе при помощи однозначных функций $u_{i}=u_{i}\left(u_{i}^{*}\right)$ (данные преобразования соответствуют калибровке каналов);

$$
\text { II : } \widehat{W}\left(u_{1}, u_{2}, u_{3}\right)=H\left(u_{1}, u_{2}, u_{3}\right) W\left(u_{1}, u_{2}, u_{3}\right),
$$

где $H \neq 0$;

$$
\text { III : } \widetilde{W}\left(u_{1}, u_{2}, u_{3}\right)=F\left[W\left(u_{1}, u_{2}, u_{3}\right)\right],
$$

где $F(0)=0, F^{\prime}(0) \neq 0$. Функцию ткани $W$ при помощи этих преобразований можно привести к каноническому виду в данной точке (см. [2]):

$$
W_{0}=u_{1}+u_{2}+u_{3}+a\left(u_{2}^{2} u_{3}+u_{3}^{2} u_{1}+u_{1}^{2} u_{2}\right)+\left(b_{1} u_{1}+b_{2} u_{2}+b_{3} u_{3}\right) u_{1} u_{2} u_{3}+\ldots,
$$


где $a=\kappa / 6, b_{j}=D_{j} \kappa / 6$; здесь $\kappa$-кривизна ткани, а $D_{j}$-инвариантные дифференциальные операторы ткани (см. [2]) в данной точке, выражающиеся через частные производные:

$$
D_{1}=\frac{\partial}{\partial u_{3}}-\frac{\partial}{\partial u_{2}}, \quad D_{2}=\frac{\partial}{\partial u_{1}}-\frac{\partial}{\partial u_{3}}, \quad D_{3}=\frac{\partial}{\partial u_{2}}-\frac{\partial}{\partial u_{1}} .
$$

В [7] подробно описан алгоритм приведения функции три-ткани к каноническому виду.

Замечание 2. Понятие канонического вида функции часто используется в теории и приложениях. Например, в теории катастроф (см. [4]) определяют и исследуют канонический вид Морса функций нескольких переменных (лемма о расщеплении), при этом допускают более общий тип замены переменных (смесь цветов):

$$
u_{1}=U_{1}\left(u_{1}^{*}, u_{2}^{*}, u_{3}^{*}\right), \quad u_{2}=U_{2}\left(u_{1}^{*}, u_{2}^{*}, u_{3}^{*}\right), \quad u_{3}=U_{3}\left(u_{1}^{*}, u_{2}^{*}, u_{3}^{*}\right) .
$$

Если эту лемму применить к функции три-ткани, то получится более грубая классификация. Как показывают примеры круговых три-тканей, одной канонической форме Морса функции триткани могут отвечать различные канонические формы Бляшке. Каноническая форма Бляшке позволяет строить новые детекторы и дескрипторы особых точек изображений, что важно при работе с большими базами изображений.

2.2. Связность ткани, поверхностный элемент и кривизна три-ткани RGB-изображения. В [2] вводятся понятия поверхностного элемента $\Omega$, связности ткани $\gamma$ и кривизны $\kappa$. Эти величины являются инвариантными характеристиками ткани трехканального изображения и определяются следующими выражениями:

$$
\begin{gathered}
\Omega=W_{1} W_{2} d u_{1} \wedge d u_{2}=\ldots, \\
\gamma=-\sum_{i=1,2,3} \frac{\partial}{\partial u_{i}} \ln \left(W_{i}\right) d u^{i}+d \ln \left(W_{1} W_{2} W_{3}\right), \\
\kappa=A_{23}+A_{31}+A_{12},
\end{gathered}
$$

где величины $A_{r s}$ имеют вид

$$
A_{r s}=\frac{1}{W_{r} W_{s}} \frac{\partial^{2}}{\partial u_{r} \partial u_{s}} \ln \frac{W_{r}}{W_{s}}=\frac{W_{r r s}}{W_{r}^{2} W_{s}}-\frac{W_{r s s}}{W_{r} W_{s}^{2}}+\frac{W_{r s}}{W_{r} W_{s}}\left(\frac{W_{s s}}{W_{s}^{2}}-\frac{W_{r r}}{W_{r}^{2}}\right) .
$$

Замечание 3. В общем случае кривизна $\kappa$ выражается через функции $u_{i}$ в виде громоздкой формулы. В частном случае, когда первые две функции имеют вид координатных функций $u_{1}=x$ и $u_{2}=y$, а третья функция $u_{3}=f(x, y)$ - произвольная три раза непрерывно дифференцируемая функция, формула упрощается (см. [5]). В данном случае функция ткани имеет вид $W\left(u_{1}, u_{2}, u_{3}\right)=f\left(u_{1}, u_{2}\right)-u_{3}$, а кривизна Бляшке три-ткани RGB-изображения определена формулой

$$
\kappa=\frac{f^{(0,2)} f^{(1,1)} p^{2}-f^{(1,2)} q p^{2}+p q^{2} f^{(2,1)}-q^{2} f^{(1,1)} f^{(2,0)}}{(p q)^{3}},
$$

где для краткости введены обозначения

$$
f^{(i, j)}=f^{(i, j)}(x, y)=\frac{\partial^{i+j} f(x, y)}{\partial^{i} x \partial^{j} y}, \quad i, j=0,1, \ldots ; \quad p=f^{(1,0)}, \quad q=f^{(0,1)} .
$$

В [7] предложена методика вычисления частных производных, основанная на переходе к дискретной сетке точек (пикселей изображения).

2.3. Круговая три-ткань $R G B$-изображения и «полная кривизна» три-ткани как дескриптор изображения. Пусть имеются два уравнения $g_{1}(x, y)=0, g_{2}(x, y)=0$, задающие окружности на плоскости:

$$
g_{1}(x, y)=a_{1,1}\left(x^{2}+y^{2}\right)+a_{1,2} x+a_{2,1} y+a_{3,3}, \quad g_{2}(x, y)=b_{1,1}\left(x^{2}+y^{2}\right)+b_{1,2} x+b_{2,1} y+b_{3,3} .
$$

Уравнение вида $u=f \cdot g_{1}+(1-f) \cdot g_{2}=0$ назовем уравнением линейного пучка окружностей с вершинами $g_{1}, g_{2}$. Разрешая равенство $u=0$ относительно $f$, получим функцию $f(x, y)$, все линии уровня которой являются окружностями. 
Определение 1 . Три-ткань $W\left(u_{1}, u_{2}, u_{3}\right)$, образованная тремя линейными пучками окружностей, называется круговой тканью.

В [3,5] дана полная классификация регулярных круговых тканей. Ниже приведены условия на функцию $f(x, y)$, при которых линии уровня прямые (или окружности).

Теорема 1 (линии уровня). Пусть $f(x, y)$ - функция класса $C^{3}$, регулярная на плоскости, m.e. $\nabla f \neq 0$. Если $C_{1}(f) \equiv 0$, то все линии уровня - прямые, а если $\left.C_{2}(f)\right) \equiv 0$, то все линии уровня - окружности, где

$$
\begin{aligned}
C_{1}(f)= & f^{(0,2)} p^{2}-2 p q f^{(1,1)}+f^{(2,0)} q^{2} \\
C_{2}(f)= & \left(p^{2}+q^{2}\right)\left(p^{3} f^{(0,3)}-3 p^{2} q f^{(1,2)}+3 p q^{2} f^{(2,1)}-q^{3} f^{(3,0)}\right)+3 f^{(1,1)}\left(q^{4} f^{(2,0)}-p^{4} f^{(0,2)}\right)+ \\
& \quad+6 p q\left(f^{(1,1)}\right)^{2}\left(p^{2}-q^{2}\right)-3 p q\left(f^{(0,2)}-f^{(2,0)}\right)\left(p^{2} f^{(0,2)}-3 p q f^{(1,1)}+q^{2} f^{(2,0)}\right) ;
\end{aligned}
$$

здесь

$$
f^{(i, j)}=f^{(i, j)}(x, y)=\frac{\partial^{i+j} f(x, y)}{\partial^{i} x \partial^{j} y}, \quad i, j=0,1, \ldots ; \quad p=f^{(1,0)}, \quad q=f^{(0,1)} .
$$

Доказательство. Пусть $\{x=x(t), y=y(t)\}$ - натуральная параметризация линии $f(x, y)=$ const, где $t$ - длина дуги. Единичный касательный вектор и единичный вектор нормали в этом случае имеют вид $\tau=\{\dot{x}, \dot{y}\}, n=\{-\dot{y}, \dot{x}\}$ соответственно (здесь точка обозначает производную по $t$ ). Из формул Френе-Серре следует

$$
\ddot{x}=-\kappa \dot{y}, \quad \dddot{x}=-\dot{\kappa} \dot{y}-\kappa^{2} \dot{x}, \quad \ddot{y}=\kappa \dot{x}, \quad \dddot{y}=\dot{\kappa} \dot{x}-\kappa^{2} \dot{y},
$$

где $\kappa-$ кривизна линии. Из четырех равенств

$$
\dot{x}^{2}+\dot{y}^{2} \equiv 1, \quad \frac{d}{d t} f(x(t), y(t)) \equiv 0, \quad \frac{d^{2}}{d t^{2}} f(x(t), y(t)) \equiv 0, \quad \frac{d^{3}}{d t^{3}} f(x(t), y(t)) \equiv 0
$$

с учетом (1) находим $\dot{x}, \dot{y}, \kappa, \dot{\kappa}$ :

$$
\kappa=\frac{C_{1}(f)}{\left(p^{2}+q^{2}\right)^{3 / 2}}, \quad \dot{\kappa}=\frac{C_{2}(f)}{\left(p^{2}+q^{2}\right)^{3}} .
$$

Условию $\kappa \equiv 0$ соответствует равенство $C_{1}(f) \equiv 0$, а условию $\dot{\kappa} \equiv 0$ соответствует равенство $C_{2}(f) \equiv 0$.

Замечание 4. При исследовании цифровых изображений возникает важная задача улучшения цифрового изображения путем удаления «шума». Естественный способ это сделать путем минимизации следующих интегральных характеристик:

$$
I C_{1}=\iint\left|C_{1}(f)(x, y)\right| d x d y, \quad I C_{2}=\iint\left|C_{2}(f)(x, y)\right| d x d y .
$$

Эти функционалы легко вычисляются для цифровых изображений при помощи разностных отношений.

\section{3. Построение круговой три-ткани и вычисление ее инвариантных характеристик.}

3.1. Построение круговой три-ткани трехканального изображения в математическом пакеme Mathematica. Рассмотрим практический пример построения конкретной круговой три-ткани в математическом пакете Mathematica. Круговая три-ткань в рассматриваемом примере будет образована тремя функциями $u_{1}, u_{2}, u_{3}$, линии уровней которых - три пучка окружностей с центрами в точках $C_{1}=(0,0), C_{2}=(0,1), C_{3}=(1,0)$ на плоскости. Значения функций меняются в пределах от 0 до 1 на квадрате $[0,2] \times[0,2]$. Функция яркости RGB-изображения в данном случае имеет вид

$$
\left\{u_{1}=\frac{\sqrt{x^{2}+y^{2}}}{2 \sqrt{2}}, \quad u_{2}=\frac{\sqrt{x^{2}+(-1+y)^{2}}}{\sqrt{5}}, \quad u_{3}=\frac{\sqrt{\left.(-1+x)^{2}+y\right)^{2}}}{\sqrt{5}}\right\} .
$$




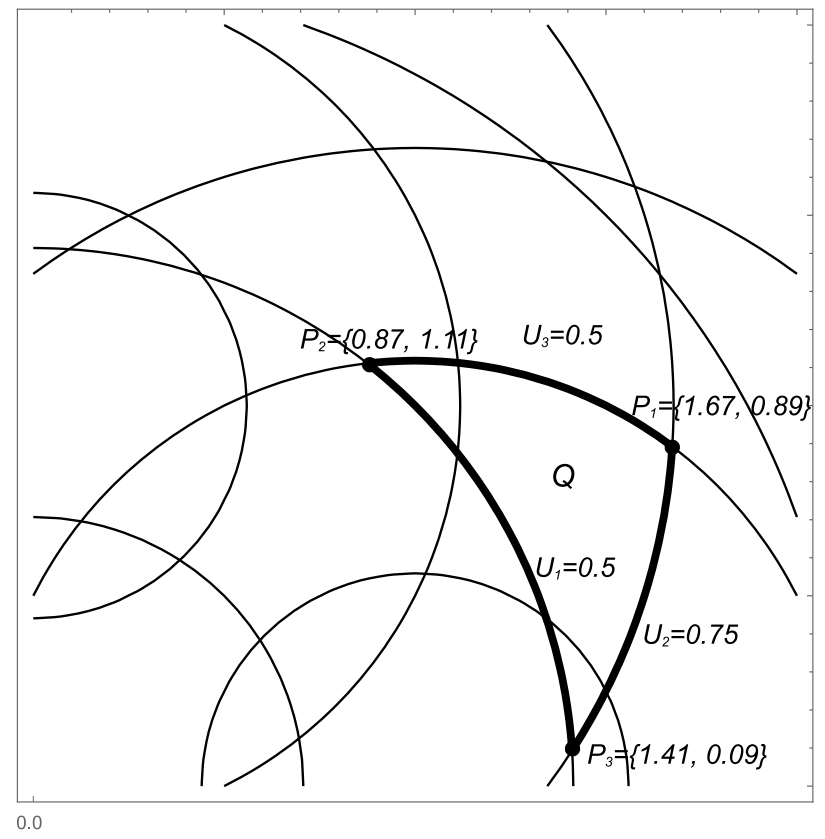

Рис. 1. Три-ткань заданного RGB-изображения

Исключая из равенств (2) координаты $\{x, y\}$, получим функцию данной три-ткани:

$$
W\left(u_{1}, u_{2}, u_{3}\right)=2+128 u_{1}^{4}-10 u_{2}^{2}+25 u_{2}^{4}-10 u_{3}^{2}+25 u_{3}^{4}-80 u_{1}^{2}\left(u_{2}^{2}+u_{3}^{2}\right) .
$$

Коэффициенты разложения канонического представления функции три-ткани позволяют без труда вычислить инвариантные характеристики RGB-изображения, в том числе связность ткани $\gamma$, поверхностный элемент $\Omega$, кривизна $\kappa$. На рис. 1 представлена соответствующая три-ткань и иллюстрация к формуле (16.6) из книги В. Бляшке «Введение в геометрию тканей»

$$
N\left(u_{1}, u_{2}, u_{3}\right)=\ln \frac{W_{1}\left(P_{3}\right) W_{2}\left(P_{1}\right) W_{3}\left(P_{2}\right)}{W_{1}\left(P_{2}\right) W_{2}\left(P_{3}\right) W_{3}\left(P_{1}\right)},
$$

которую можно использовать как «метку» или дескриптор изображений.

3.2. Вычисление инвариантных характеристик для круговой три-ткани в среде MatLab. Для вычисления инвариантных характеристик круговой три-ткани авторами работы разработан программный модуль в среде Matlab. Он представляет собой набор функций по вычислению и анализу таких инвариантных характеристик трехканального изображения как связность ткани $\gamma$, поверхностный элемент $\Omega$, кривизна $\kappa$. Дополнительно для оценки вычисленных характеристик производится отображение гистограммы распределения значений вычисленной характеристики или графика значений характеристики. Также, по выбору пользователя, производится вычисление корреляции между полученными характеристиками.

4. Заключение. Предложенный в работе подход к анализу изображений, основанный на теории три-тканей В. Бляшке, является актуальным и перспективным направлением в области обработки цифрового изображения. Требующими внимания, на наш взгляд, являются задачи выявления особых точек трехканального изображения в терминах уравнения ткани, поиск интегральных характеристик три-ткани изображения и локальных топологических признаков изображения, определение локальной корреляции двух изображений или, другими словами, близости двух слоений на плоскости.

Представленные методы построения три-ткани цифрового трехканального изображения (в том числе и круговой три-ткани), а также определения и вычисления инвариантных характеристик три-ткани цифрового изображения могут найти применение в решении широкого класса задач 
обработки, анализа и классификации изображений, поиска соответствия по образцу. В ходе разработки программного модуля в среде Matlab были реализованы эффективные алгоритмы вычисления различных топографических характеристик (атрибутов) цифрового изображения. Программный модуль в системе Matlab может быть использован как в дальнейших исследованиях по обработке цифровых изображений, так и в учебных целях.

\section{СПИСОК ЛИТЕРАТУРЫ}

1. Базылев В. Т. О многомерных сетях и их преобразованиях// в кн.: Итоги науки. Геометрия. 1963.. М.: ВИНИТИ АН СССР, 1965. - С. 138-164.

2. Бляшке В. Введение в геометрию тканей. - М., 1959.

3. Лазарева В. Б. Классификация регулярных круговых три-тканей с точностью до круговых преобразований// Фундам. прикл. мат. - 2010. - 16, № 1. - С. 95-107.

4. Постон Т., Стюарт И. Теория катастроф и ее приложения. - М.: Мир, 1980.

5. Шелехов А. М., Лазарева В. Б., Уткин А. А. Криволинейные три-ткани. - Тверь, 2013.

6. Samarina O. V. Group Images Invariants. — Lambert Academic Publishing, 2010.

7. Samarina $O . V$., Slavsky V.V.W. Blaschke's theory application in digital image processing// J. Math. Sci. Appl. - 2013. - 1, № 2. - P. 17-23.

8. Xia G. S., Delon J., Gousseau Y. Locally invariant texture analysis from the topographic map// Proc. 19th Int. Conf. on Pattern Recognition (Tampa, Florida, USA, December 8-11, 2008). - Paris, France, 2009. P. 1-4.

Самарина Ольга Владимировна

Югорский государственный университет, Ханты-Мансийск

E-mail: samarina_ov@mail.ru

Семенов Сергей Петрович

Югорский государственный университет, Ханты-Мансийск

E-mail: ssp@ugrasu.ru

Славский Виктор Владимирович

Югорский государственный университет, Ханты-Мансийск

E-mail: slavsky2004@mail.ru 